\title{
Fatiguing Data to Protect against Cyber Security Extortions: A counter- intelligence methodology
}

\author{
Dr. Anthony Vincent B \\ Assistant Professor, Department of Computer Science \\ Kristu Jayanti College (Autonomous) \\ K. Narayanapura, Kothanur(PO), Bangalore - 77 \\ anthonyvincent@kristujayanti.com
}

\begin{abstract}
"Now and recently, confab is less about preventing and stopping an attack, threat or exposure, and more about how swiftly you can detect that an attack is happening." There's a growing demand for security information and event management (SIEM) technologies and services, which gather and analyse security event big data that is used to manage threats. Big data offers the ability to analyse immense numbers of potential security events and make connections between them to create a prioritized list of threats. With big data, distinct data can be connected, which allows cyber security professionals to take a proactive approach that prevents attacks. Advanced Persistent Threats (APTs) are also used to find and identify where threats are coming from. Integrated security architecture and power of automated information collection and sharing between many security systems, called "Counter-intelligence" to solve the strategic short comings. "Counter intelligence" translates to new security product architecture into a data collection backbone feeding a centralized repository used to correlate security anomalies from, across multiple systems. This paper illustrates the new counter intelligence approach to defend against future cyber security threats by applying modern risk analysis and mitigation methods to protect users' private data from big data.
\end{abstract}

Keywords: Big data, Cyber security, APT, Counter intelligence, SIEM, 


\section{Introduction}

Cybercrime costs $\$ 118$ billion annually and takes an average of 18 days to resolve at a cost of nearly $\$ 416,000$ over those 18 days - and those figures are expected to grow as cyber-attacks continue to increase. Fortunately, tools and techniques now exist to handle the volume and complexity of today's cyber-attacks, enabling enterprises to stay ahead of evolving threats. [2] Combining big data analytics with security technologies yields a stronger defence posture. Big security analytics provide highspeed, automated analysis to bring network activity into clear focus to detect and stop threats, and shorten the time to remediation when attacks occur.

\section{Big data analytics in Cyber Security}

Cyber security teams today typically use multiple endpoint solutions to protect their enterprises from common cyber threats. Each tool generates alerts based on a particular kind of suspicious activity. But none of these tools is equipped to detect sophisticated, adaptive attacks - the kind of attacks that target the world's largest and most critical institutions on a daily basis. There are many services available that specialize in taking multiple sources of threat intelligence and trying to make sense of all the data by using advanced techniques to correlate and find causation of cyberattacks. The web is rich with signals of data breaches, information about newly vulnerable targets, and evidence of pre-planned attacks, but it's nearly impossible to organize all of this information with manual or ad-hoc systems. [4]

Big Data Cyber Security Analytics systems specialize in discovering sophisticated attack patterns against an organization, even when those attack patterns do not occur frequently or with an obvious pattern. Big Data Cyber Security Analytics systems or advanced customized software defined networking defence solutions provides a richly collaborative environment in which analysts can employ successful investigative strategies developed by their peers. Analysts can also track how cyber 
threats change over time and pre-emptively mitigate threats they have seen before. Security teams can spindle from passive alert processing to proactive threat detection and counter-intelligence. Recognizing that commercial institutions face a shared set of cyber threats, Big Data Cyber Security Analytics is about creating a platform for secure information sharing across organizational boundaries that can help to strengthen and set this understanding, and expose unforeseen challenges. Providing critical intelligence to gain deeper insight utilized in further defensive, detection, analytical, and investigative activities. [7]

\section{Cyber Counter-Intelligence: A main layer}

Cyber-crimes and Cyber warfare activities have been gaining unprecedented momentum over the past few years. Driven by criminally or politically motivated individuals, groups and organizations, they pose a threat to the IT and Web infrastructures of governments, corporations and even private individuals worldwide. The mitigation of Cyber threats, like any other, is based on a multi layered approach. In the Cyber warfare arena, the obvious defence layer is that of technology - installing firewalls, switches and sniffers. The most overlooked layer is the Intelligence layer knowing your Cyber foes and exactly what threats they pose. Intelligence as a concept is viewed as a very broad term, mostly associated with military affairs. Today's Cyber battlefield has evolved to become very similar to a "classic terror" battlefield, with similar intelligence needs and benefits. Most of the actors in the Cyber arena are either non-state actors or state sponsored actors. These activists and units do communicating and thriving "live" on the internet while targeting mostly non-military targets. [5]

By utilizing a similar approach to counter-terror related intelligence, our approach provides relevant and actionable intelligence on the activities, capabilities and motivation of Cyber criminals and hacktivists threatening IT assets, infrastructure and interests. Our unique approach to Cyber intelligence relies on penetrating the online 
Cyber networks where Cyber activists motivate, plan and carry out Cyber-attacks and develop new techniques and technologies. By becoming part of the Cyber-crime network, our counter intelligence provides unprecedented insight into the activities and capabilities of Cyber activists around the world. [6]

\section{Our cyber services include:}

- Mapping of Players- Hackers, hacktivists, terror groups and more

- Broad coverage spectrum- hackers forums, closed groups, Darknet

- Identification of MO and Attacking Tools

- Identification and analysis of relevant cross-industry threat trends

- Analysis of Past Cyber Attacks

- Assessment of Potential Threats

- Online alerting regarding future attacks and consulting for threat mitigation

\section{Defensive Cyber Counterintelligence}

Defensive CCI can be understood of as actions taken to identify and counter adversary intrusions before they occur as well as the efforts in identifying and minimizing the threat landscape. The intent of Defensive CCI is to understand the adversary and minimize the threat landscape to which they might exploit; the product of this effort is usually reports and analyses that defenders can use to complement their overall personnel, network, and information security. One of the most performed Defensive CCI actions is a red team assessment. Think of the team that is tasked to perform a network assessment to determine where weak points exist and where an adversary might gain access to information systems. The red team must have an understanding of adversary tactics, techniques, and procedures to accurately act like the adversary. [1] In essence, the red team helped identify the threat landscape to the organization and inform the organization on how they could reduce it. 


\section{Offensive Cyber Counterintelligence}

Offensive CCI can be understood of as interactions with the adversary to directly collect information about their intelligence collection operations or to deceive them. Offensive CCI can be leveraged in a number of ways including the use of sock puppets or fake personas on online forums to gather information about adversary intelligence collection operations such as capabilities, victims, tactics, etc. the flipping of adversary operators into double agents to infiltrate the adversary's operation, or in publishing false reports and information to deceive adversary intrusion attempts. These efforts can be performed both inside and outside of your networks. For example, an Offensive CCI operation could be run to identify or mitigate adversaries already in your network. [1] An Offensive CCI team could help create a honeypot inside your network to identify malicious actors on the network.

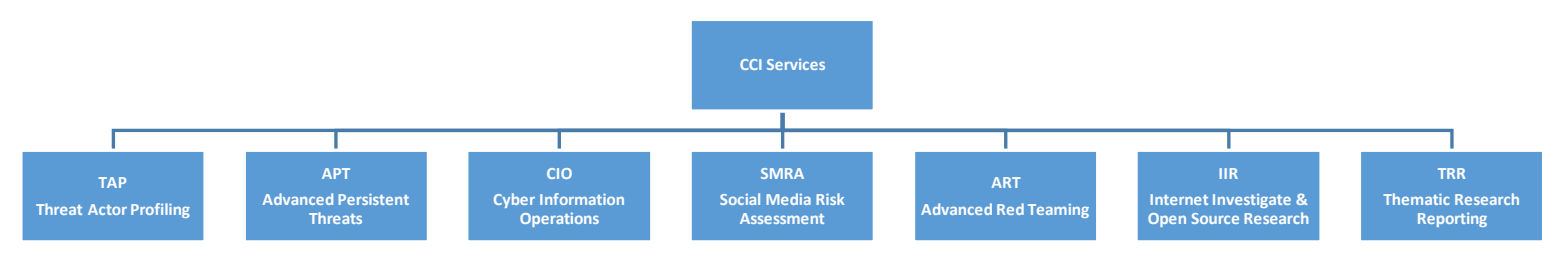

Figure 1: Counter Intelligence Services

\section{Counter Intelligence Services:}

1. Threat Actor Profiling (TAP) - understanding the 'who' of the five Ws (what, when, were, why) is a critical component of effectively assessing the threat that an opposing group presents to a company. 
2. Advanced Persistent Threat (APT) - APT is an umbrella term used to describe the cyber portion of an on-going foreign intelligence gathering campaign; whereby increasingly sophisticated cyber threats seek to gain/maintain network access and collect intellectual property, personally identifiable information, and financial and/or strategic information from governments, corporations and individuals.

3. Cyber Information Operation (CIO) - CIO is a process of promoting a positive message about a Client over a negative messaging regarding a Client. Simply put the CIO service creates a greater positive narrative about the Client, than the negative narrative that is being created by other Internet users.

4. Social Media Risk Assessment (SMRA) - the ubiquity of social media in the work place has meant that it is becoming harder than ever for security teams to track employee social media use and the ways proprietary data may be flowing out of the Business..

5. Advanced Red Teaming Penetration Testing (ART) - we have the ability to simulate the threat of a range of cyber threat actors. From highly technical cyber espionage actors to the disruptive antics of 'script kiddy' activists, it has the ability to actively Penetration Test your infrastructure according to the threat posture of a large number of threat actor types.

6. Internet Investigation and Open Source Research and Analysis (IIR) - with the increasing spread of cyber space and the ubiquity of personal data on the Internet, for the wise researcher the Internet can prove to be a gold mine of valuable information for individuals with the skill and time to mine this source.

7. Thematic Research Reporting (TRR) - taking a deep dive look at strategic topics a TRR examines security issues that will affect a company over a protracted period of time. 


\section{Conclusion}

Big data will have an impact that will change most of the product categories in the field of computer security including solutions, network monitoring, authentication and authorization of users, identity management, fraud detection, and systems of governance, risk and compliance. Big data will change also the nature of the security controls as conventional firewalls, anti-malware and data loss prevention. In coming years, the tools of data analysis will evolve further to enable a number of advanced predictive capabilities and automated controls in real time. Our proposal of this paper is a turnkey solution provider for Cyber counter intelligence needs. Our research assessment process continuously monitors Cyber Threat developments and provide our customers with intelligence-based threat alerts and analysis. Working with industry leading cyber security partners, we are able to complement these alerts with solid technical consulting to countenance appropriate threat mitigation.

\section{Future Enhancement}

By 2016, more than $25 \%$ of global firms will adopt big data analytics for at least one security and fraud detection use case, up from current $8 \%$. Big data analytics gives enterprises faster access to their own data than ever before. Big data analytics enables enterprises to combine and correlate external and internal information to see a bigger picture of threats against their enterprises. It is applicable in many security and fraud use cases such as detection of advanced threats, insider threats and account takeover. [3]

Organizations should align the capabilities security in a holistic cyber security strategy tailored to the threats and the risks specific to the demands of the organization, big data requires the collection of information from various sources and in different formats, a logical target is to have a single architecture to collect, index, normalize, analyse and share all the information, and organization should look for profile accounts, 
users or other entities, and look for anomalous transactions against those profiles. Organizations should ensure that the continued investment in security products promote technologies that use approaches agile-based analysis, not static signature-based tools to threats or on the edge of the network .Organizations are more than ever exposed to a large number and variety of threats and risks to cyber security. Big Data will be one of the main elements of change in the enterprises by supplying intelligence-driven models. Big data analytics will play a crucial role in detecting crime and security infractions in future cyber-space.

\section{References}

[1] Sean Bodmer, Gregory Carpenter, Lance James \& David Dittrich , 2004, 'Hacking Back: Offensive Cyber Counterintelligence', Paperback, First Edition.

[2] Michael Minel, Michele Chambers \& Ambiga Dhiraj, 2013, 'Big Data, Big Analytics: Emerging Business Intelligence and Analytic Trends for Today's Businesses', Wiley Publications, Hardcover.

[3] Frank J. Ohlhorst, 2013, 'Big Data Analytics: Turning Big Data into Big Money', Wiley \& SAS Business Series.

[4 Big data: Cyber security's silver bullet. Available from < $\underline{\text { http://www.forbes.com/sites/kurtmarko/2014/11/09/big-data-cyber-security/>.[11 }}$

Sep 2014]

CSC: $\quad$ Cyber

Security

Solutions

<http://www.csc.com/cybersecurity/publications/>.[3 Mar 14]

[6] Terrogance Web Intelligence <http://www.terrogence.com/capabilities/cybercounter-intelligence/>.[Since 2013]

[7] Big data cyber security Analytics in Action<http://www.drchaos.com/big-datacyber-security-analytics-in-action/>.[25 Aug 2014] 